\title{
Residual stresses around welds in nickel-based
}

\section{superalloys}

Alexander M. Korsunsky, Karen E. James

Dept of Engineering Science, University of Oxford, Parks Road, OX1 3PJ, UK

Short title: $\quad$ Residual stresses in nickel superalloys

Contact author: $\quad$ alexander.korsunsky@eng.ox.ac.uk, fax +44 (1865) 273010

\begin{abstract}
Welding of nickel-based superalloys is employed in the manufacture of many important components of the jet engine, such as combustor casings, compressor disc assemblies, etc. Welds induce microstructural modification in the weld seam and the heat affected zone (HAZ) which generally reduce the fatigue resistance compared to that of the parent metal. Welds also generate significant residual stresses, both locally due to combination of solidification shrinkage and high temperature plastic deformation, and also globally through the general tensioning of the structure. Diffraction of high energy synchrotron $\mathrm{X}$-rays is very well suited to the task of accurate non-destructive evaluation of residual stresses near welds in thin walled components assembled by welding nickel superalloys. Residual stresses induced by linear and annular welds produced in test pieces made from C263 and IN718 nickel based alloys under different conditions are evaluated, and the effects of various heat treatments on stress state are discussed.
\end{abstract}

Keywords: welding, residual stress, synchrotron X-ray diffraction 


\section{INTRODUCTION}

The primary source of concern about the presence of residual stresses is associated with component distortion which may lead to the violation of the tight tolerance limits, and so require expensive rework or scrap. The quantification of residual stresses is also required for lifing calculations.

The magnitude of residual stresses in welded nickel-based superalloy assemblies are best characterised experimentally using diffraction of penetrating radiation, such as neutron beams or high-energy X-rays [3]. The penetration of such beams into the material exceeds that of laboratory X-rays by several orders of magnitude, and thus allows the evaluation of bulk stresses, or stress resultants in thin-walled plate and shelllike structures. Furthermore, comparative analysis of strains in crystallites of certain orientation (i.e. reflection-specific strains) reveals information about sample deformation history, e.g. plastic strain [4].

In the present paper several sets of measurements carried out using high energy synchrotron X-ray diffraction are described. These include electron beam welds (EBW) and gas tungsten arc welds (GTAW), including the special case of low stress, no distortion (LSND) welds. The test pieces were C263 and IN718 flat plates and annular test samples. Comparisons are made with some measurements carried out using neutron diffraction instruments.

\section{RESULTS AND DISCUSSION}

\section{C263 Plates}


Rectangular $100 \times 50 \times 2 \mathrm{~mm}$ C263 test pieces contained $80 \mathrm{~mm}$ long autogenous gas tungsten arc welds. SXRD measurements were performed on the ID11 beamline using the KUMA diffractometer using incident X-ray wavelength of $0.2529 \AA$ (49.15keV). Inplane components of strain longitudinal and transverse to the weld line were measured. The longitudinal strain component calculated in the basis of the 111 peak is shown in in Figure 1, together with neutron diffraction results from the D1A instrument (ILL), where the same reflection was used [1]. The general agreement between these two different techniques is clearly very good.

Figures 2a and 2b show the comparison, on the same scale, of the longitudinal strains in a regular weld with those in an LSND weld. Strains shown were calculated using different reflections (111, 200 and 311). These reflections display different response to inelastic deformation, and therefore not always suitable for macrostress measurement. However, it follows from the same argument that the difference between the strains measured using different reflections contains information about the inelastic strains. This has been confirmed theoretically and experimentally [4].

Figure 2 demonstrates that significant variation of the 'difference strain' between reflections 111 and 200, say, occurs near the weld line, confirming that the inelastic strains are localised in this region. It is also apparent that LSND treatment resulted in a significant reduction in the residual stress magnitude.

\section{IN718 Test Pieces}

Electron beam welding was used to manufacture two kinds of welds. Firstly, linear welds were produced in flat rectangular flat plates (Figure 3a). Secondly, flange-andweb annular test pieces were manufactured containing two concentric circumferential welds (Figure 3b). SXRD measurements of in-plane residual strains were performed on 
the ID11 and ID31 beamlines at the ESRF using monochromatic mode and a scanning diffractometer [2]. Under the assumptions of plane stress the in-plane stress components were calculated, designated longitudinal (along the linear weld) and transverse (perpendicular to the linear weld) in the rectangular pieces, and radial and hoop in the annular pieces, respectively.

It is well know that thermal processes such as welding result in the redistribution of alloying elements, and may lead to significant changes in the unstrained lattice spacing. For all measurements discussed in this section unstrained lattice spacing values $\mathrm{d}_{0}$ for each reflection were determined as follows. Identical welded pieces were cut by electric discharge machining (EDM), so as to produce thin $(\sim 1 \mathrm{~mm})$ and long $(\sim 30 \mathrm{~mm})$ matchstick samples from different areas of the weld. Stresses in a small volume $\left(\sim 1 \mathrm{~mm}^{3}\right)$ at the end of each matchstick are substantially relaxed by cutting. Therefore diffraction patters collected from these gauge volumes were assumed to correspond to the strain-free condition, and provides $\mathrm{d}_{0}$ values for strain evaluation in the same region.

In the rectangular welded plate the stresses were evaluated along several lines running at different distances from the plate edge perpendicular to the linear weld (Figure 4). The set of measurements provides insight into the amount of constraint offered by the material surrounding the source of inelastic strain in the weld, and the relief provided by the free edge. The measurement results compared well with a qualitative model of the weld line as a distribution of strain nuclei.

The second set of measurements in IN718 pieces was carried out on a series of test specimens containing two concentric circumferential welds that attach an annular web to thicker inner and outer flanges. This test specimen shape is representative of the constraint exerted on the thin-walled shell-like structures by bosses and similar thicker wall elements often used at critical locations. The continuous circular nature of the weld 
results in an increased constraint and a higher level of residual stresses. Strain measurements were made along radial lines running across the web between the inner and outer flange in test pieces subjected to different heat treatments.

Very significant tensile stresses (in excess of 500MPa) were observed in the web. Experimentally this was found to lead to increased stiffness of the assembly as a whole (the 'drum' effect). An explanation for this structural effect can be derived by considering small deflection of the specimen under axial displacement of the inner flange, and taking into account the effect of tensile residual stress in the web.

The influence of these treatments on the stress state is illustrated in Figure 5. It is clear that solution heat treatment is required in order to effect a significant reduction in the residual stress level; however, this may be incompatible with the treatment schedules required by other parts of the assembly. It is apparent from the results that ageing treatment alone is insufficient for stress relief. This is perhaps not entirely surprising, for two reasons. Firstly, ageing is accompanied by material strengthening, which does not encourage residual stress reduction by plastic flow. Secondly, the level of constraint imposed on the membrane by the solid flanges results in a kinematic constraint, which cannot be relieved without substantial deformation. Solution treatment temperatures are required to achieve this result.

\section{CONCLUSION}

The use of high energy synchrotron X-ray diffraction provides an excellent tool for stress analysis around welds in nickel superalloys, and can well serve the purposes of both non-destructive evaluation and model validation. Further research effort in this area needs to be directed at the provision of instruments and facilities capable of accepting and manipulating real large scale engineering components. 


\section{REFERENCES}

1. D. Dye, K. T. Conlon, R.C. Reed, K.E. James and A.M. Korsunsky, Diffraction strain measurements for the characterization of residual stresses in welds for gas turbine applications, Trends in Welding Research (2002).

2. M.V.R.S. Jensen, D. Dye, K.E. James, A.M.Korsunsky, S.M. Roberts and R.C. Reed, Residual stresses in a welded superalloy disc: Characterization using synchrotron diffraction and numerical process modeling., Metall. Mater. Trans., A33, 2921-2931 (2002).

3. Korsunsky, A.M., K.E. Wells, and P.J. Withers, Mapping two-dimensional state of strain using synchroton X-ray diffraction. Scripta Materialia, 39, 1705-1712 (1998).

4. Korsunsky, A.M., M.R. Daymond, and K.E. Wells, The development of strain anisotropy during plastic deformation of an aluminium polycrystal. Mater. Sci. Forum, 347-3, 492-497 (2000). 


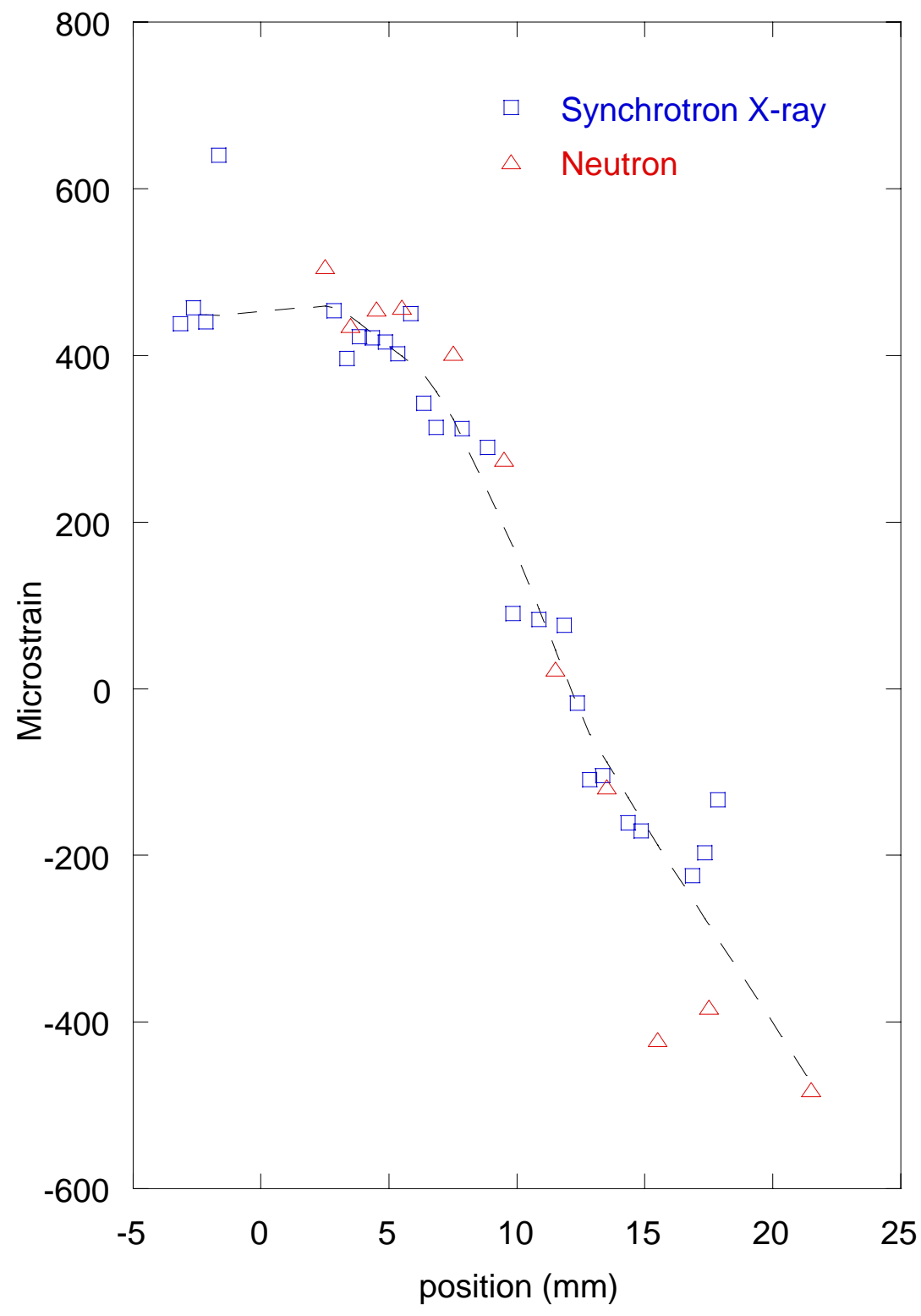

Figure 1. Comparison of the longitudinal strain in the C263 weld measured by SXRD and neutron diffraction. 


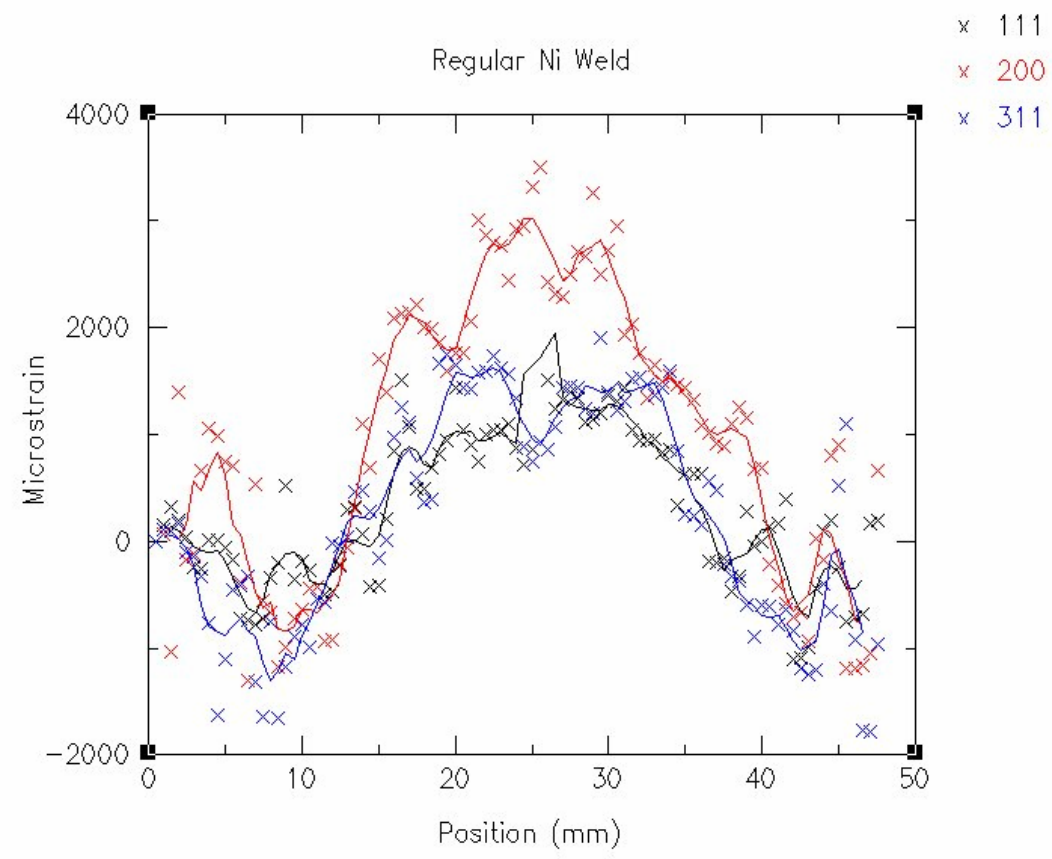

(a)

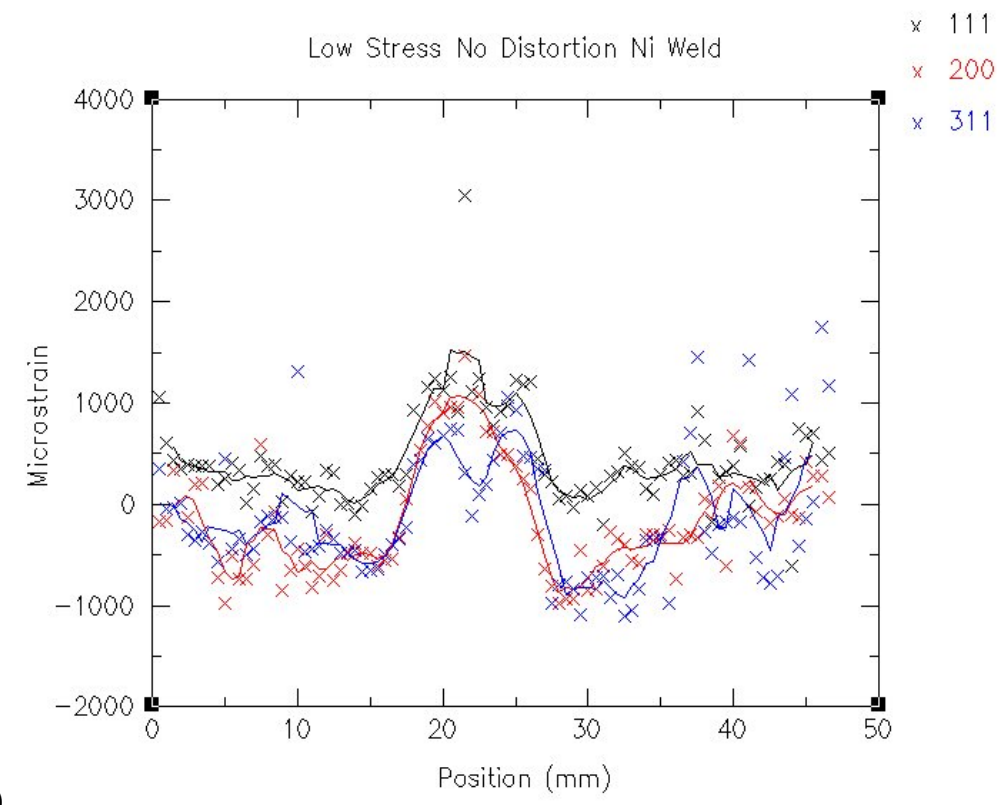

(b)

Figure 2. Comparison of the residual longitudinal strain in a regular weld (a) and an LSND weld (b). The vertical scale is kept the same for clarity of comparison. 

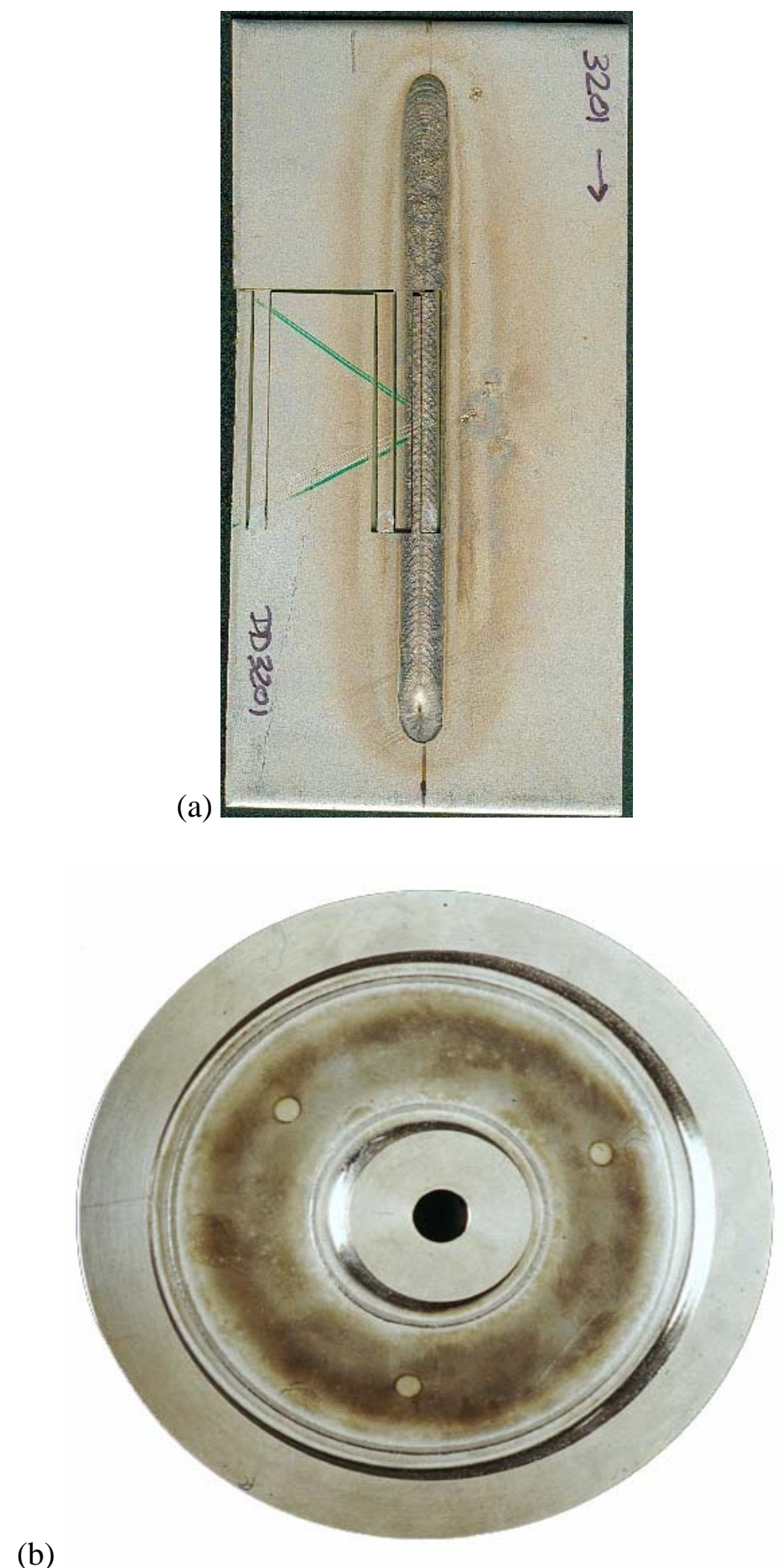

(b)

Figure 3.Test pieces containing (a) linear and (b) circumferential EB welds. 


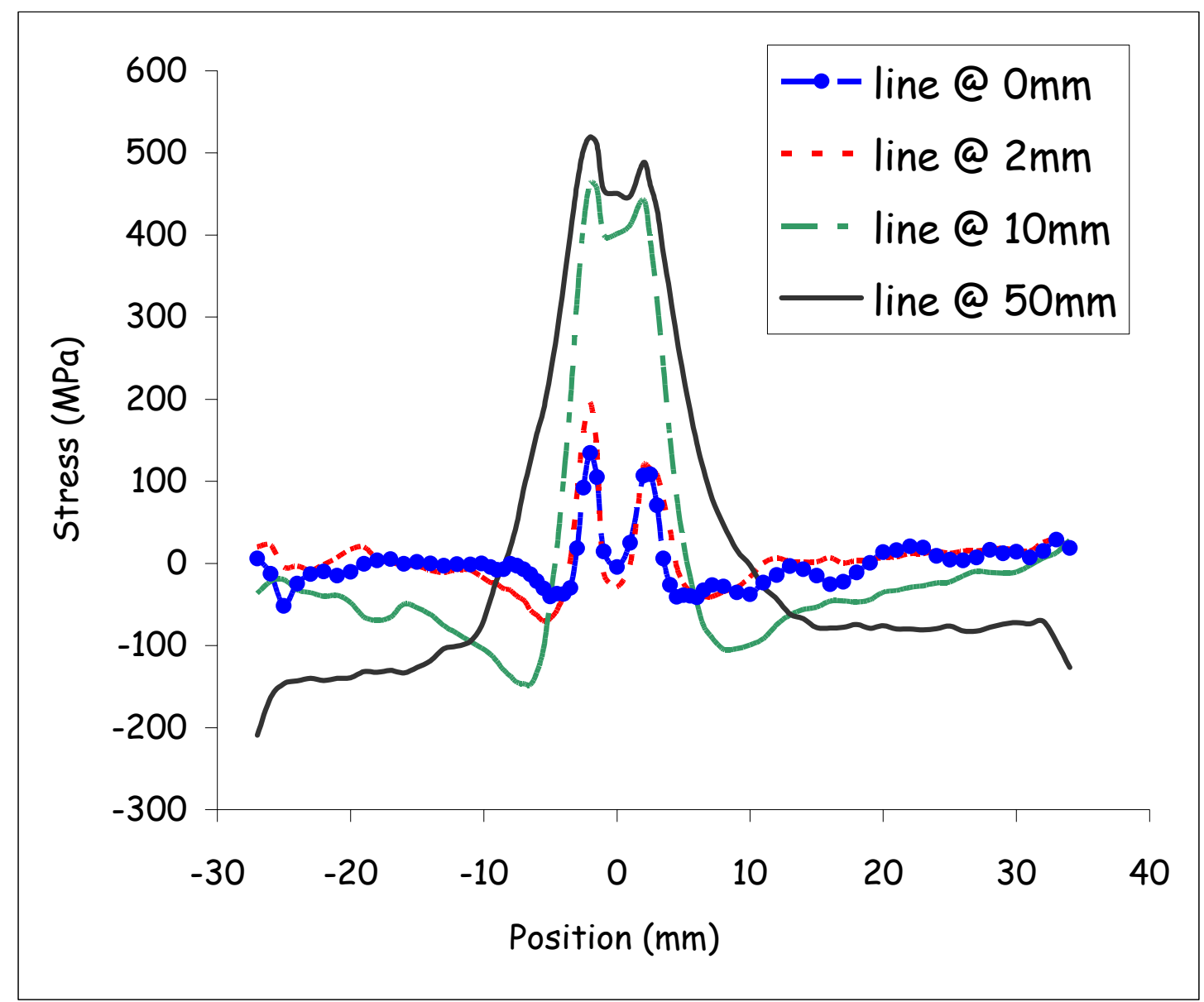

Figure 4. Effect of edge relief on magnitude of residual stress along a straight weld line, shown as a function of the distance from the plate edge. 


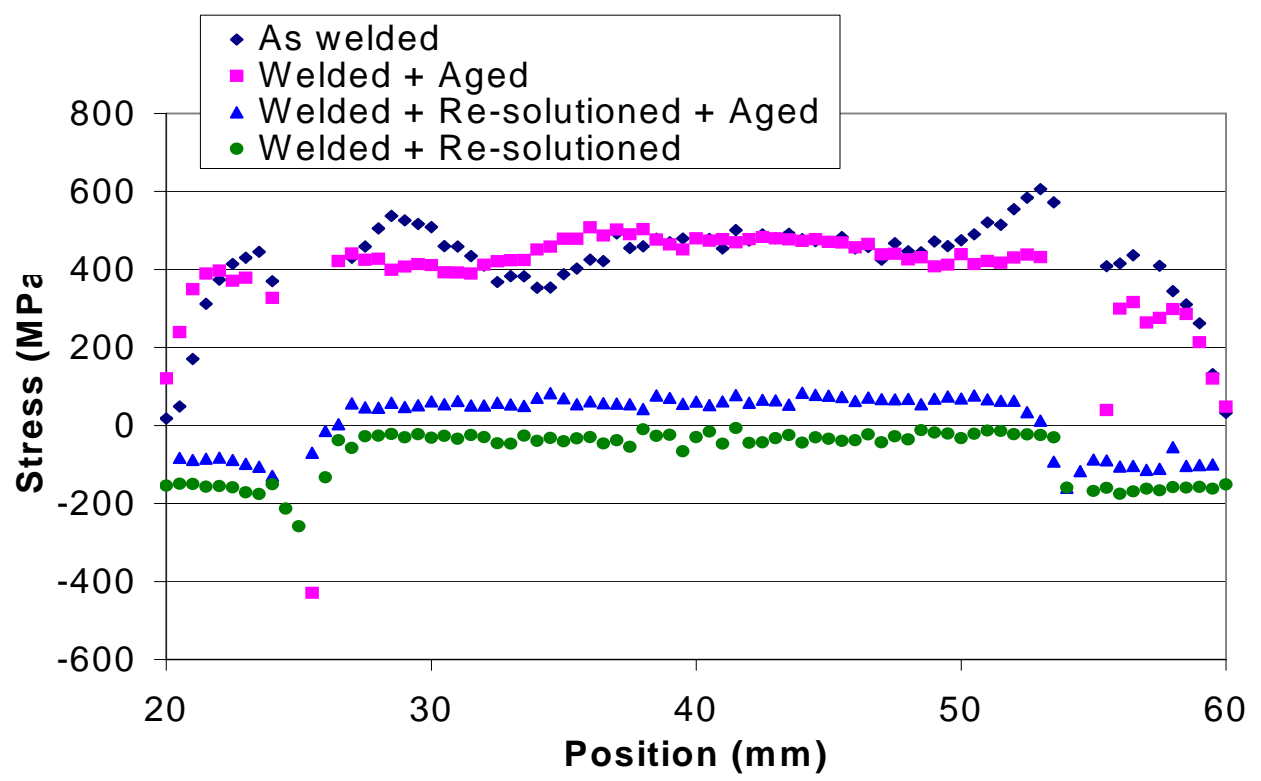

Figure 5. Effect of solution and ageing heat treatments on the residual hoops stresses in annular IN718 test pieces . 